\title{
Psychiatric assertive outreach and learning disability services
}

\section{Angela Hassiotis, Peter Tyrer \& Patricia Oliver}

\begin{abstract}
Assertive outreach is a well-established method of managing patients with severe mental illness in the community. However, there is limited application and evidence of its efficacy in services for people with learning disabilities who also have mental illness. This paper elucidates current service provision for this group of patients and illustrates the pathways to mental health care available to them.
\end{abstract}

Learning disability ('mental retardation' in ICD-10 and DSM-IV) is characterised by global developmental delay, an IQ below 70 and deficits in adaptive skills. It has been shown that adults with learning disabilities on the one hand can experience any of the psychiatric problems that affect the general population and on the other, have higher rates of mental disorders compared with counterparts of average ability. Rates vary for different disorders, but schizophrenia in particular appears to be three times more common in people with learning disabilities. Comparative studies of patients with schizophrenia, with and without learning disability, indicate that the adults with learning disability suffer with more negative symptoms, impairment of episodic memory, a history of epilepsy and social isolation (Doody et al, 1998). They also more frequently have family histories of learning disability and/or schizophrenia. In addition, brain scans of people with learning disability and comorbid mental illness show higher rates of structural abnormalities.

A longitudinal study of a British birth cohort showed that adults with mild learning disability are four times more likely to have affective disorders not accounted for by medical conditions or social disadvantage than is the general population (Richards et al, 2001). A recent epidemiological survey suggested that phobic disorders are also more common in individuals with learning disability (Deb et al, 2001). However, the study excluded rates for dementia, challenging behaviour and autism. Another epidemiological study (Cooper, 1997) showed rates for dementia to be higher in the learning-disabled population over 65 years of age without Down's syndrome compared with the general population (16\% v. $5 \%$ ).

Such associations were acknowledged rather late, in the wake of the closure of institutions and the advent of community care for people with learning disabilities. Initially, the general opinion was that all challenging behaviours and mental disorders would cease to exist outside the institutional facilities, a social policy tenet particularly prevalent in the UK and the USA. Until the early 1970s, funding for the development of mental health services for people with learning disabilities was unavailable and the assumption was that generic mental health services should undertake the treatment of such patients who required specialist mental health service provision. Current government policy (Department of Health, 2001) also maintains that mental health services for the general population should be accessible by adults and older people with learning disability who have mental health problems. Therefore, people with learning disabilities and their families should now be exposed to interventions such as assertive outreach, crisis resolution and alternatives to admission to inpatient psychiatric units.

Assertive outreach was developed at the same time as the programme of deinstitutionalisation of

Angela Hassiotis is a clinical senior lecturer in the psychiatry of learning disabilities and an honorary consultant psychiatrist at Royal Free and University College Medical School (Department of Psychiatry and Behavioural Sciences, Wolfson Building, 48 Riding House Street, London W1N 8AA, UK. E-mail: a.hassiotis@ucl.ac.uk). Her main research interest is the evaluation of mental health services and interventions for people with learning disabilities and she also has been involved in the development of a memory service for older people with learning disabilities. Peter Tyrer is Professor of Psychiatry at Imperial College Faculty of Medicine and Editor of The British Journal of Psychiatry. His main research interests are in evaluations of mental health interventions and personality disorder. Patricia Oliver is a research associate in learning disabilities at the Department of Psychological Medicine, Imperial College Faculty of Medicine. Her main research interests are in the evaluation of mental health services and interventions for people with learning disabilities. 
people with mental illnesses which took place in the 1970s. It was introduced in the US state of Wisconsin in the 1970s because of frustration with the lack of community service provision for people with severe mental illness at that time (Stein \& Santos, 1998). It was a precursor of case management, as a guarantor of continuity of care and support to people with mental health problems living in the community. Case management promoted coordinated services intended to be efficient and effective, although this has been contradicted by evidence from controlled trials (Marshall et al, 1995; Marshall, 1996). Assertive community treatment (the word 'outreach' is a UK addition) was conceived as a service model that could reduce admissions to hospital of severely mentally ill patients and, unlike case management, there is a good evidence base for this claim (Marshall \& Lockwood, 2002).

Teams that practised assertive outreach 'went out to the patient' and actively sought to maintain contact and continuity of care for those unlikely to engage with standard services (e.g. by attending outpatient appointments). The current version of assertive outreach, where delivered, includes small case-loads (fewer than 15 patients per case manager), a multi-disciplinary framework, treatment in the patient's home and assertive follow-up. Case managers are also able to support carers and perhaps offer more specific interventions. There are several criticisms of 'assertive community treatment' and 'intensive case management' that centre on the fidelity of the model delivered. Assertive outreach, an expensive high-resource intervention, is best suited to those with severe mental illness, comorbid substance misuse and those who are heavy service users, but the evidence for its value comes almost entirely from the USA and has not been replicated in the UK (Harrison-Read et al, 2002).

\section{Learning disabilities and assertive outreach}

\section{The evidence so far}

There has been only tentative implementation of the assertive outreach model in mental health services for people with learning disability, and that only in the past 5 years or so. Research of the efficacy of assertive outreach for the population with learning disabilities as well as mental disorders has been scant. Van Minnen et al (1997) randomly allocated 50 patients with severe mental illness and mild learning disability to either outreach/community treatment or to standard treatment - that is, hospital admission. Patients were followed up for 7 months. The treatment team consisted of a psychiatrist, an educationalist, a social worker, three community psychiatric nurses and a coordinator. The programme was based on a keyworker system, but the team helped to devise and review the care plan. Despite methodological limitations of a small sample and short follow-up time, the study showed that the patients in the outreach arm of the trial had significantly fewer admissions, there was no increase in carer burden and costs were lower overall.

In a randomised controlled trial, intensive case management (fewer than 15 patients per case-load) was found to significantly reduce the number of admissions of patients with borderline or mild learning disabilities and severe mental illness; improve the reported unmet needs; increase patient satisfaction; and reduce the cost of in-patient health care (Burns et al, 1999; Hassiotis et al, 2001). These findings were of particular interest because those without a learning disability in the study did not benefit from intensive case management (Burns \& Guest, 1999).

\section{Clinical experience}

\section{USA}

Meisler et al (2000) described the use of the assertive community treatment model in the treatment of patients with mild learning disabilities and mental health problems living in the community. The programme was originally developed following a class action lawsuit in the State of North Carolina, USA. Twenty-one patients participated in the programme for a year, but the available data have reported on only 10 of these. Apart from mental disorders, some of the patients had histories of arson, self-injury and assault. The designated team offered psychiatric evaluation and treatment, substance misuse treatment, training in activities of daily living and social skills, occupational opportunities and assistance with practical tasks. The assertive community treatment model was also used to decrease the amount of supervision, in some cases on a 24-hour basis, for some of the patients who were receiving treatment. Benefits for the participants included an increase in total days in employment (1926 v. 273), reduction in hospital days (38 v. 187) and shorter duration of admissions by about 8 days. In addition, costs for those in the assertive community treatment programme fell by $15 \%$ (US\$168 000 v. US\$198 000).

However, despite the positive results of the model in terms of its impact on community integration and self-development of the participants, it had to be abandoned owing to demands to further reduce expenditure. 
UK

Learning disability services in the UK have traditionally been built around the core element of 'community learning disability teams' - the equivalent of the community mental health teams in adult mental illness that have a good record of achievement in severe mental illness (Simmonds et al, 2001). These teams have maintained a generic focus on treatment rather than solely targeting individuals with mental health problems. Although this model has helped to foster the unique multi-disciplinary nature of community learning disability teams, it has also led to a stalemate in the growth of appropriate interventions and a shortage of skilled professionals to treat and maintain people with mental health problems, particularly in the community.

Existing community learning disability teams are multi-disciplinary and health-oriented, and they include several professionals who do not have mental health as a priority or indeed may not have any training in mental health. In fact, the mental health component is considered to be only a small part of the diverse work that the teams usually undertake. This ranges from assessments for wheelchairs and home adaptations, environmental risk assessment, psychometric evaluation, dysphagia assessments, training and systemic consultations, clinical work, physiotherapy input and health promotion; all members of the teams also act as informal advocates for their clients (Box 1).

Often there are tensions between the team members, some of whom may feel that overemphasising mental health problems is stigmatising to the majority of clients, who need support by a specialist team but who do not have mental disorders. At the present time, most of the teams are moving into joint working with social services, and several learning disability services have been transferred into primary care trusts, although there is wide variation in the organisational configurations chosen in different parts of England at least.

Box 1 Roles of the community learning disability team

- Assessment of health and social care needs

- Assessment of skills and the environment

- Speech and language therapy

- Occupational opportunities

- Community support

- Support with medication and hospital appointments

- Drop-in service for social security, finances, housing

- Physiotherapy assessment and treatment

- Psychotherapeutic interventions
Cases referred to the psychiatrists in the teams are more likely to be placed under the care programme approach (CPA) and to receive additional help from community psychiatric nurses and psychologists, particularly if they also exhibit challenging behaviour. The CPA care coordinator takes a more active role in maintaining contact with the patients, although there is no strict reinforcement of a 'must go to the patient' policy. However, CPA is not a core service for several learning disability services.

\section{Examples of assertive outreach}

A few community-based learning disability services have begun to address the issues of intensive support for patients with mental health problems, but they have come up with various different approaches. Some teams follow the 'team within a team' model. For example, in several London boroughs (e.g. Brent, Harrow, Barnet and Waltham Forest) such teams practice a version of assertive outreach, i.e. a few chosen professionals (psychiatrist, psychologist and behavioural specialist nurse) from the larger community learning disability team work intensively with patients with challenging behaviour.

Other services adopt the 'distinct assertive team' model, which has several configurations. For example, they might have a single team that includes a consultant psychiatrist and other health professionals such as psychologists and nurse specialists in challenging behaviour (e.g. North East Essex and its Clinical Specialist Team); or a separate team comprising occupational therapists/nurses who have a small case-load and work intensively with clients and led by a psychologist (e.g. the Support and Management Team in the boroughs of Ealing and Hounslow).

City and Hackney Primary Care Trust Services for People with Learning Disabilities has an assertive outreach team with dedicated staff, supported by a psychiatrist and a psychologist. Some may have been admitted to local mental health hospitals or are in out-of-borough placements; often they are detained under the Mental Health Act 1983. Many fail to engage voluntarily with services and are at risk of self-neglect or harm (for interventions see Box 2). Contact with the patient is described on the patient information leaflet as 'frequent'.

This confusion is clearly illustrated by the difficulties of undertaking a randomised controlled trial of assertive outreach for adults with learning disability and mental health problems in London. Oliver et al (2002: p. 341) write:

\footnotetext{
'At present, given the diverse service configurations it was difficult to find an operational definition that would ensure valid treatment to those randomly allocated. The agreed proposal was that frequency
} 
Box 2 Interventions practised by an assertive outreach team

The assertive outreach team in Hackney, London, offers the following support to people with learning disabilities:

- Assessment of care

- Skills teaching (e.g. daily living skills)

- Risk assessment and CPA

- Psychological support (e.g. cognitivebehavioural therapy)

- Medication advice and monitoring

- Evaluation of physical health

- Help with access to education and employment

- Relapse prevention

- Regular reviews

and types of contact should be compared, but not team structure, which had been an integral part of the mental health trials. It is possible to randomise referrals into two treatment groups, an "assertive" group that received visits from more than one professional more than once a week and a "standard" group that received visits from one professional no more than once a week. This reflected actual practice in most teams, despite lack of clarity as to why some people got more resources than others did.

Despite those problems, Oliver et al reached an agreement with four teams (three in London and one outside) to carry out a pilot study that is expected to provide further evidence for the efficacy of assertive community treatment in learning disability mental health, the results of which will become available shortly. The aim of the project was to investigate the effect of frequency of contact on the number and duration of hospital admissions, quality of life, carers' well-being and service costs.

Community-based interventions distinct from residential care provision have been tried, with some success, for people mainly presenting with challenging behaviour and severe learning disability. Such teams are 'psychology-led' and called 'peripatetic. Their main purpose is to maintain patients in community homes, and behavioural interventions are the mainstay of treatment. However, the outcomes have been modest, for example patients appear to benefit from increase in meaningful engagement with the carers and improved quality of daytime occupation. Problem behaviours were unaffected (Hassiotis, 2002). However, many of these studies are very small in scope, uncontrolled and offer little description of the team's working routine.

Another example of a type of assertive outreach is provided by the Camden Learning Disabilities Service and the Islington Learning Disability Partnership. Community support workers (without mental health training) from the social-services-led Community Support and Outreach Team operate a support system that complements the main aspects of the individual's care plan and can visit patients in their homes more than once a week if necessary. For patients who are living independently, they offer telephone contact and visits outside of normal office hours during the week and similar support via a duty system at weekends. They provide assistance with activities of daily living and some monitoring of mental state and medication, although they are not directly involved in administering the latter. Community support workers - and community psychiatric nurses - will assist the patient with encouragement to take medication, facilitating the receipt of prescriptions and dosette boxes, explaining how to take medication, its side-effects and counting tablets. Members of the team who are keyworkers for particular patients attend ward rounds for them if they are in-patients and liaise with other mental health teams regarding discharge planning. They also work with the nursing staff in the hospital to organise leave and to escort their patients to appointments or to occupational and leisure opportunities. The following vignettes show examples of how the Camden Learning Disability Service is working with patients with mental illness.

\section{Case vignette 1}

Ms H. is a 58-year-old woman who has been discharged to the community from an institution. She lives in a warden-controlled flat with her partner of many years, whom she met at the institution. They both have mild learning disabilities, but Ms $\mathrm{H}$. is more able than her partner, who has serious physical problems. The couple do not trust professionals, tend to leave their home early in the morning, return late in the afternoon and unhook the phone so that they cannot receive any calls. Ms $\mathrm{H}$. has a history of severe depression with psychotic features and does not take medication. Since her admission to the local in-patient unit, she is being supported more intensively by the community learning disability services, who had also worked with her during the admission. She has since accepted minimal help in dealing with benefits and the help of a community psychiatric nurse regarding her medication and health appointments. She has managed to cope with an episode of ill health in her partner and was seen daily at the hospital rather than at home as she was hardly ever there. She takes her medication, which is monitored and put in a dosette box weekly, and has even attended an out-patient clinic.

\section{Case vignette 2}

Ms G. is a single woman in her 50 s with a long history of paranoid schizophrenia, who has had three inpatient admissions in the past 15 months. During her most recent admission, she was prescribed clozapine and supported by both the nursing staff and her care 
coordinator in discussing her options about medication, understanding the symptoms that may indicate relapse (distress and persecutory delusions about the police and staff), the proposed treatment and the side-effects. Ms G. has been in contact with the care coordinator/keyworker at least weekly and with other members of the team for such things as day activities and cognitive-behavioural therapy for her delusions. She has been attending a day centre successfully (she had had no activities up to 8 months before her recent admission) and has been on day trips with staff and clients from the centre.

If there are concerns about the mental state of any patient in either Camden or Islington who is known to the boroughs' general health services, care managers or Community Support and Outreach Team, a 'virtual team' of staff from both boroughs is convened so that a quick plan of action can be implemented. These virtual teams have been introduced to ensure a seamless transition from inpatient to community services, and ward staff work alongside community staff in a joint team which plans together the patients' discharge and rehabilitation.

In the event of a crisis, the members of the virtual team can respond to the acute needs of the individual, liaise with other professionals and temporarily increase input to the individual.

Where appropriate, the keyworkers will make referrals to the local mental health crisis teams for further home support, especially if an admission might be considered at any stage. Although there are designated beds for patients with learning disability in one of the adult psychiatric wards, if they are full then the admission occurs under the care of the sector consultant psychiatrist.

\section{What next?}

According to the National Service Framework for Mental Health, crisis teams should be available in all adult mental health services to provide community or home treatment to patients with acute mental illness. These teams should also be accessible to dually diagnosed adults with mild or moderate learning disabilities where mental health problems are the central problem. Using the principles of health facilitation (Department of Health, 2002), the community learning disability team should jointly co-work such cases in order to ensure that there is continuity of care. Furthermore, patients should also be able to access other innovative mental health services such as the first-episode psychosis and crisis teams.

Unfortunately, in many learning disability services, organisational changes have occurred with little consultation with the clinicians, which has increased, in many instances, the isolation of already highly pressed services and has led to only limited access to mainstream provision. As a result, conflicts between mental health and learning disability services, poor management of patients, and lack of coordination of discharge and after-care have been reported.

It is questionable whether it is necessary to duplicate efforts in establishing assertive outreach teams specifically for people with learning disabilities, or indeed whether such intervention should only be offered to people with severe mental illness and mild learning disability (Hassiotis et al, 2001). Perhaps a solution might be to develop specialist teams within learning disability services for those who have enduring mental illness, frequent hospitalisations, episodes of deliberate self-harm and lack of engagement with the community services. Such patients may also have substance misuse problems and, occasionally, forensic histories. It could be argued that certain aspects of the input by many community learning disability teams resembles an assertive outreach model, i.e. increasing contact frequency for limited periods when it is required, administration of medication and monitoring of mental state, and promoting coping skills. It is, however, still a long way away from the real establishment of a shared approach to planning community care for patients with mental illnesses.

\section{References}

Burns, T. \& Guest, L. (1999) Running an assertive community treatment team. Advances in Psychiatric Treatment, 5, 348356.

-, Creed, F., Fahy, T., et al (for the UK700 Group) (1999) Intensive versus standard case management for severe psychotic illness: a randomised trial. The Lancet, 353, 21852189.

Cooper, S. A. (1997) Epidemiology of psychiatric disorders in elderly compared with younger adults with learning disabilities. British Journal of Psychiatry, 170, 375-380.

Deb, S., Thomas, M. \& Bright, C. (2001) Mental disorders in adults with intellectual disability. I: Prevalence of functional psychiatric illness among a community based population aged between 16 and 64 years. Journal of Intellectual Disability Research, 45, 495-505.

Department of Health (2001) Valuing People: A New Strategy for Learning Disability for the 21st Century. London: Stationery Office.

- (2002) Action for Health: Health Action Plans and Health Facilitation. Detailed Good Practice Guidance on Implementation for Learning Disability Partnership Boards. London: Stationery Office.

Doody, G. A., Johnstone, E. C., Sanderson, T. L., et al (1998) 'Pfropfschizophrenie' revisited. Schizophrenia in people with mild learning disability. British Journal of Psychiatry, 173, 145-153.

Harrison-Read, P., Lucas, B., Tyrer, P., et al (2002) Heavy users of acute psychiatric beds: randomised controlled trial of enhanced community management in an outer London borough. Psychological Medicine, 32, 413-416.

Hassiotis, A. (2002) Community mental health services for individuals with intellectual disabilities: issues and 
approaches to optimising outcomes. Disease Management and Health Outcomes, 10, 409-417.

- Ukoumunne, O., Byford, S., et al (2001) Intellectua functioning and outcome of patients with severe psychotic illness randomised to intensive case management: report from the UK700 trial. British Journal of Psychiatry, 178, $166-171$.

Marshall, M. (1996) Case management: a dubious practice. $B M J, 312,523-524$.

— \& Lockwood, A. (2002) Assertive community treatment for people with severe mental disorders. Cochrane Library, issue 2. Oxford: Update Software.

—, Lockwood, A. \& Gath, D. (1995) Social services casemanagement for long-term mental disorders: a randomised controlled trial. The Lancet, 345, 409-412.

Meisler, N., McKay, C., Gold, P. B., et al (2000) Using principles of ACT to integrate community care for people with mental retardation and mental illness. Journal of Psychiatric Practice, 6, 77-83.

Oliver, P. C., Piachaud, J., Done, J., et al (2002) Difficulties in conducting a randomized controlled trial of health service interventions in intellectual disability: implications for evidence-based practice. Journal of Intellectual Disabilities Research, 46, 340-345.

Richards, M., Maughan, B., Hardy, R., et al (2001) Longterm affective disorder in people with mild learning disability. British Journal of Psychiatry, 179, 523-527.

Simmonds, S., Coid, J., Joseph, P., et al (2001) Community mental health team management in severe mental illness: systematic review. British Journal of Psychiatry, 178, 497-502.

Stein, L. I. \& Santos, A. B. (1998) Assertive Community Treatment of Persons with Severe Mental Illness. London and New York: WW Norton.

Van Minnen, A., Hoogduin, C. A. \& Broekman, T. G. (1997) Hospital vs. outreach treatment of patients with mental retardation and psychiatric disorders: a controlled study. Acta Psychiatrica Scandinavica, 95, 515-522.

\section{Multiple choice questions}

1 ACT is an established model of treatment for:

a people with severe learning disabilities and challenging behaviour

$\mathrm{b}$ adults with severe mental disorders

c older patients with mental illness who are not engaging with services

d adults with mild learning disabilities and severe mental illness

e adolescents with substance misuse problems.

2 Assertive community treatment in learning disability services:

a is an integral part of community care

$\mathrm{b}$ can be given by untrained careworkers

c emphasises engagement with the service

d can improve the patients' daytime activities

e focuses on increased contact with patients.
3 Learning disability services:

a have a wider remit than mental health alone

b offer 24-hour support

c promote access to adult mental health facilities for their patients

$\mathrm{d}$ provide a range of interventions

e selectively treat severe mental illness.

4 Research into assertive community treatment offered to people with learning disabilities and mental disorders shows that:

a it is cheaper than standard care

$\mathrm{b}$ it results in better clinical outcomes

c patients are unhappy with it

d it reduces patients' unmet needs

e it reduces the time that patients spend in psychiatric hospitals.

5 Mental health learning disability services:

a maintain regular contact with patients when they are admitted to hospital

b will offer care to patients who do not have severe mental illness

c can provide behavioural interventions

d monitor medication daily

e have an open referral system. 